\title{
Determination of Curvature by an Osculometer
}

\author{
By Harvey L. Curtis
}

\begin{abstract}
The experimental determination of the curvature at a point of a plotted curve can be made by means of an osculometer, which is a series of arcs of known curvature. One use of such information is in the computation of the acceleration of a body for which a displacement-time curve has been constructed. By this method the acceleration can be obtained with the same accuracy as by double differentiation without the necessity of plotting the velocity time curve.
\end{abstract}

\section{Introduction}

The curvature of a curve at any point is defined as the reciprocal of the radius of the osculating circle at that point. If the equation of the curve is known, the curvature can be determined by analytical methods. However, the equations of most experimental curves are not known, so that a graphical method of determining the curvature is sometimes useful. The osculometer is a device for graphically determining the curvature at a given point on a curve by comparison with a series of ares of known curvature. Its greatest usefulness is when the data are so plotted that the radius of curvature at points to be measured is more than $10 \times 10^{-3}$ reciprocal centimeters.

\section{Types of Osculometers}

Two types of osculometers have been developed, the single-line osculometer and the double-line osculometer. The single-line osculometer shown in figure 1 consists of a series of arcs of circles, which are uniformly distributed along the axis of the osculometer and which have uniformly increasing curvatures. The centers of the circles of which the arcs are a part all lie in the axis. For general use, the simple osculometer consists of black lines on the lower surface of a rectangular sheet of transparent material. For convenience in use, the axis of the osculometer is midway between the long sides of the rectangle. The paper on which is drawn the curve to be studied is laid on a flat surface and a point on the curve selected at which the curvature is to be determined.

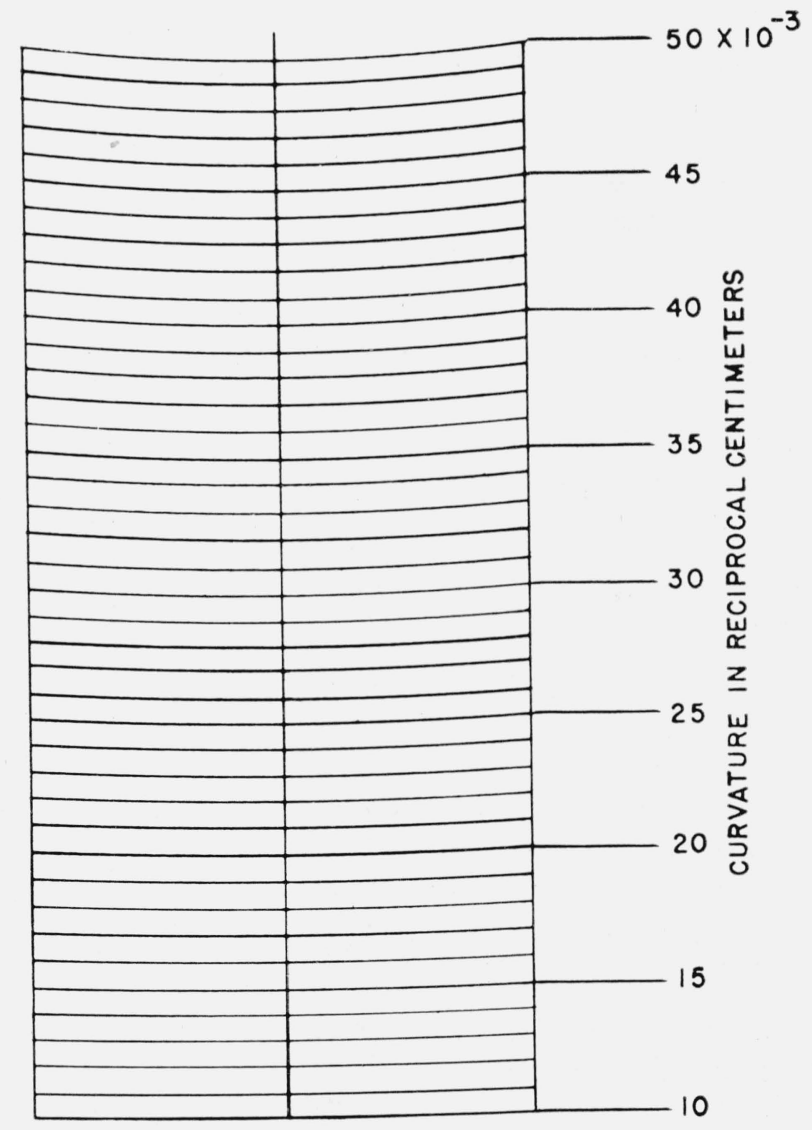

Figure 1. Single-line osculometer, solid arc.

The osculometer is placed over the curve with its axis normal to the curve at the selected point. It is then moved axially until there is found an arc that fits the curve at that point. The curvature is then read from the scale of the osculometer. 
The instrument as described has the disadvantage that, when the are is fitted, it may obscure the curve being studied. Two methods of avoiding this difficulty while using a single line osculometer have been used. In the first, the arcs were made as clear lines on an opaque or semi-opaque surface. The curve to be studied was then drawn on translucent paper and the comparison made on a diffusing surface that was illuminated from underneath. Another way of overcoming the difficulty was to make each arc as a series of dashes.

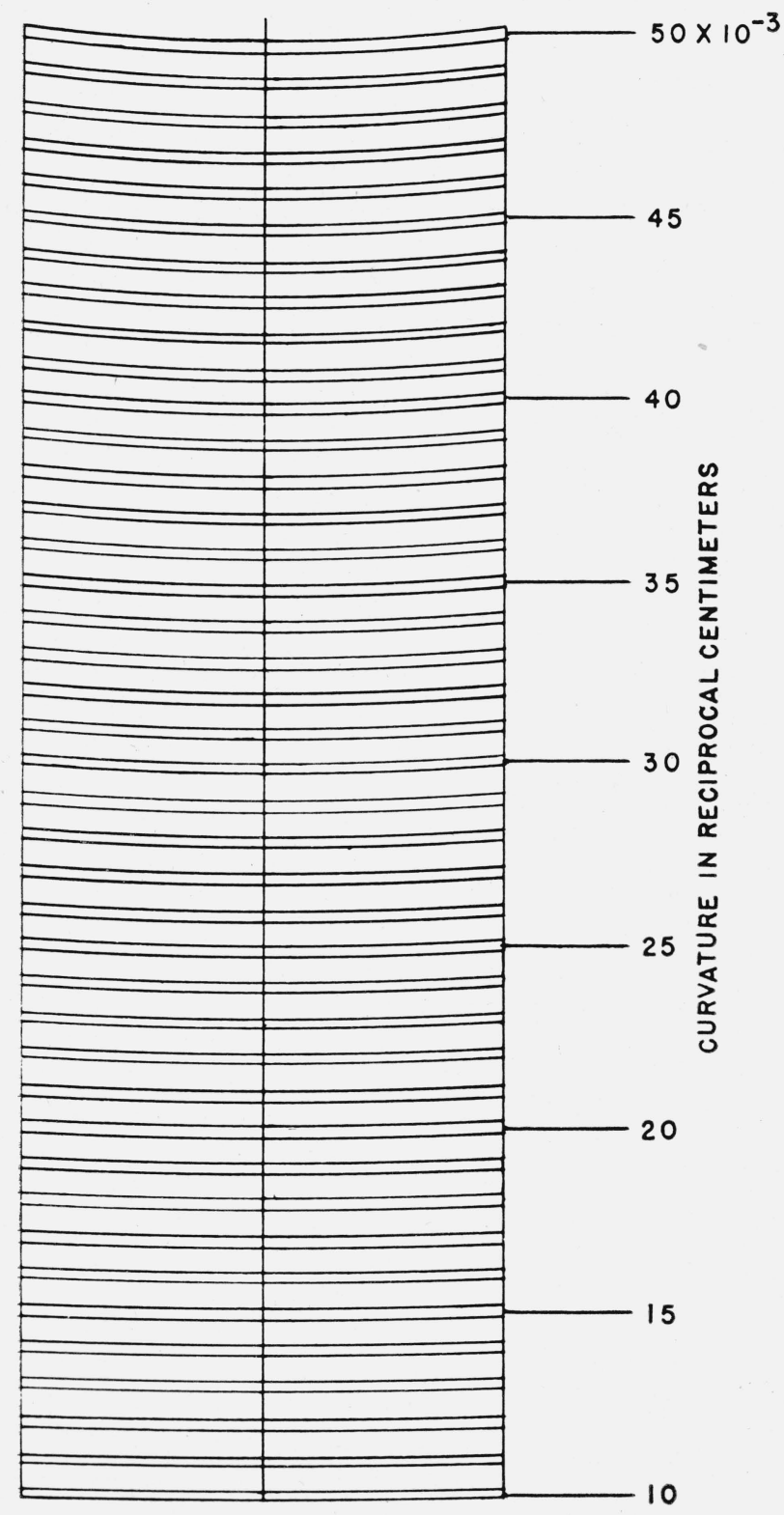

Figure 2. Double-line osculometer.
The curve then was seen between the dashes. This scheme may be advantageous with either the dark line or the bright line instrument. Other modifications can readily be devised.

The double-line osculometer shown in figure 2 was developed so that the observed curve would at all times be visible. Two arcs of the same curvature are drawn, the distance between them being about $1 \mathrm{~mm}$. The distance between successive members of the series is 3 or $4 \mathrm{~mm}$, so that the different members stand out distinctly from the individual pairs. In use, the curve to be studied is made to lie between the two curves having the same curvature as shown in figure 2 . With such an instrument, the curvature at a point can usually be determined to $1 \times 10^{-3}$ reciprocal centimeters, provided the curvature is approximately constant for a distance of $2 \mathrm{~cm}$ on both sides of the point that was selected. Some operators find the double-line osculometer easier to use than the single-line instrument. Any difference in accuracy has not been demonstrated.

In constructing either type of instrument the lines are first ruled on a transparent sheet of plastic, such as Plexiglass. The rulings are then transferred to a photographic plate by contact printing. This gives an accelerometer with black lines on a clear surface. A print of this accelerometer gives clear lines on a black surface.

\section{Use of the Osculometer in Determining Acceleration}

By using an osculometer, the acceleration of a body at any time or position can be obtained directly from its displacement-time curve, which is the plot of time as abscissae with displacements as ordinates. A point on this curve is selected at which a value of the acceleration is desired. An osculometer is selected that has an arc that will fit the curve at the selected point. When an arc has been fitted to the curve at that point, the curvature, $C$, is read directly from the osculometer and the slope, tangent $\theta$, is determined from a measurement with a protractor of the angle that the base of the osculometer makes with the $x$-axis.

The acceleration, $\alpha$, is equal to $\frac{d^{2} s}{d t^{2}}$, and hence is proportional to $\frac{d^{2} y}{d x^{2}}$, so that the equation for computing it can be derived from the expression for 
the radius of curvature, $\rho$, given in all texts on differential calculus, viz.:

$$
\rho=\frac{\left[1+\left(\frac{d y}{d x}\right)^{2}\right]^{3 / 2}}{\frac{d^{2} y}{d x^{2}}} .
$$

\section{Man:}

In this equation $\rho, x$ and $y$ are all expressed in the same units. It follows since since $\frac{d y}{d x}=\tan \theta$ that $\frac{d^{2} y}{d x^{2}}=[1 / \rho]\left[1+\tan ^{2} \theta\right]^{3 / 2}=C \sec ^{3} \theta$, where the curvature, $C$, is defined as $1 / \rho$. Applying this to a displacement-time curve, in which the measured displacements are designated as $s$ and the measured times as $t: y=r s$, and $x=r V_{0} t$, where $r$ is the constant by which a unit displacement is multiplied to give a plotting unit, and $V_{0}$ is the velocity by which a unit of time is multiplied to give unit measured length. Then

and

$$
\frac{d s}{d t}=V_{0} \frac{d y}{d x} .
$$

$$
\frac{d^{2} s}{d t^{2}}=r V_{0}^{2} \frac{d^{2} y}{d x^{2}}
$$

and therefore

$$
\alpha=\frac{d^{2} s}{d t^{2}}=r V_{0}^{2} C \sec ^{3} \theta,
$$

where $C$ is the curvature in the reciprocal of the length unit of the coordinate system. If the osculometer is calibrated in a unit different from the unit of the coordinate paper, the observed curvature must be multiplied by that conversion factor, which changes a length from the osculometer units to the coordinate units. Thus, if the osculometer is graduated in reciprocal centimeters and the coordinate unit is inches, the observed curvature, $C_{0}$, must be multiplied by 2.54 to obtain the curvature, $C$, in the coordinate system.

\section{Accuracy Attainable in Measuring Acceleration}

The accuracy attainable depends both on the accuracy of measuring the slope and on the accuracy of determining the curvature. By taking the logarithmic derivative of the equation:

$$
\alpha=r V_{0}^{2} C \sec ^{3} \theta
$$

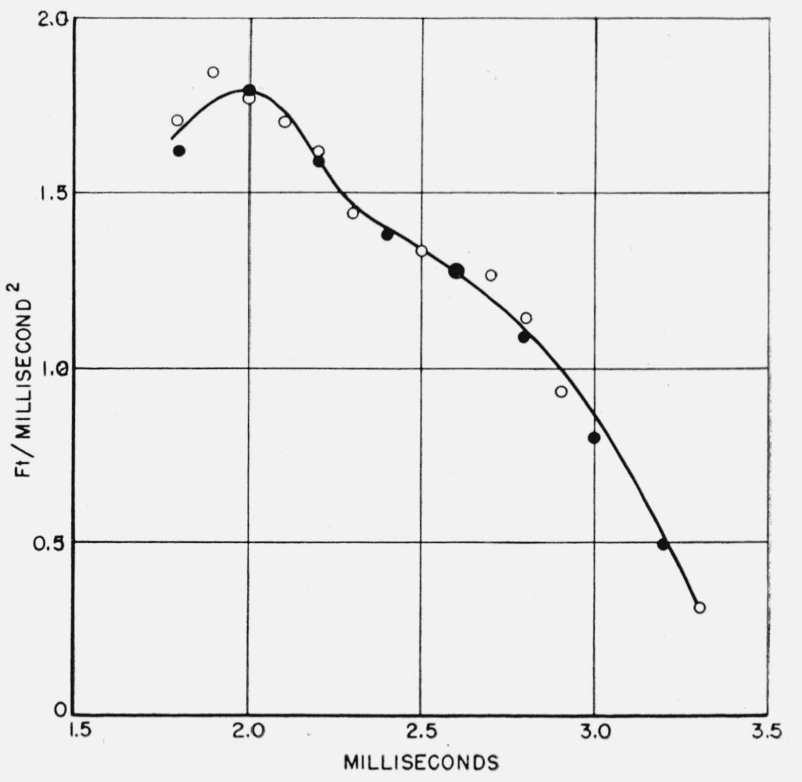

Figure 3. Comparison of results obtained on the same displacement time curve by the osculometer method and by double graphical differentiation.

, Acceleration by osculometer; $\bullet$, acceleration by double differentiation..

we obtain

$$
\frac{d \alpha}{\alpha}=\frac{d C}{C}+3 \tan \theta d \theta
$$

This equation gives the error in the acceleration as the sum of accidental errors in the curvature and slope.

For small angles, $\tan \theta$ is small, so that an error, $d \theta$, in measuring the slope may produce negligible effect in the acceleration. Then the relative error $d C / C$ in obtaining the curvature produces the same relative error in the acceleration. Thus if the displacement-time curve has a maximum or minimum, the accuracy at these points depends solely on the accuracy of the curvature determination. In general, the error in the slope measurement does not predominate until $\theta$ is more than $45^{\circ}$, but becomes excessive as $\theta$ approaches $90^{\circ}$. Hence for determination of the acceleration by an osculometer, the displacement-time curve should be plotted to such a scale that at no point does the slope exceed $45^{\circ}$. On the other hand, the plotting should not be such that the curvature is so small that it cannot be determined with reasonable accuracy. In some cases it may be desirable to plot different parts to different scales. For example, if a body starts its motion from rest and 
continually accelerates at an increasing rate, the most precise values of acceleration can be obtained by plotting the early part of the motion with a more condensed time scale than is used during the latter part of the motion.

An experimental determination of the accuracy obtained in measuring acceleration by means of an osculometer was obtained by comparing the results from an observed displacement time curve with the results obtained from the same data by the conventional method of using double differentiation. In the conventional method, the velocity of the accelerating body is obtained at a large number of points from the measurement at each point of the angle which a tangent to the displace- ment-time curve makes with the $X$-axis. Then a velocity-time curve is plotted, from which the acceleration is obtained at any point by a measurement of the angle that a tangent to the curve makes with the $X$-axis. The results by the two methods for a particular set of observations are shown in figure 3 . The curve was drawn to approximate the mean of all the points. The results by double differentiation lie, on the average, somewhat nearer the curve. If only acceleration is required, the added labor of constructing the velocity curve will seldom be justified.

Washington, August 18, 1949. 